\title{
PROBLEMS OF SENSORY PROSTHESIS
}

\section{NORBERT WIENER}

This is an age in which a great deal is being done to help the handicapped. There is an improvement in artificial limbs for the maimed, in new sorts of spectacles, and in hearing aids. All this is returning to useful life a considerable part of those who would in other generations have been hopelessly handicapped. Even the two greatest handicaps from which humanity suffers-blindness and deafness-are the object of powerful and determined attacks with the object that where these defects cannot be removed or corrected by medical means, these victims may still be allowed to take, as nearly as possible, a normal part in life.

Notice that these two afflictions are sensory defects, that is, defects in the reception of impressions from the outer universe and from other human beings. From the standpoint of the outer universe, blindness is overwhelmingly the greater of the two losses. The sense of hearing, as brought out by such investigations as have been made by the Bell Telephone Company in their study of speech, and as further evidenced by the size of the speech areas in the brain, is a sense with vastly less variety than the sense of sight. On the other hand, normal communication between man and man goes by mouth and ear much more than by all other channels, and the social and emotional damage done by deafness is disproportionately great when one compares it with the social loss of the blind. The typical emotional picture of the deaf man is that of a reserved, self-contained, neurotic personality, whereas the typical picture of the blind man who has achieved any degree whatever of equilibrium is that of a euphorically confident and rather cocky personality.

In order to make any effort to replace the lost sense, either of the blind or of the deaf, it is necessary for us to make a rather accurate measure of what they have lost. It is then necessary for us to see if there are any relatively unused channels into the human nervous system which are capable of supplying the whole or any considerable part of what is lost. These are problems of physiology and communication engineering, and have a most important mathematical side.

To bring out this mathematical side adequately, I wish to refer to

The twenty-third Josiah Willard Gibbs Lecture, delivered at New York City, December 28, 1949, under the auspices of the American Mathematical Society; received by the editors April 10, 1950. 
communication mechanisms which are more accessible to us in their whole than the human nervous system. A very interesting type of machine which is engaged in continual conversation with its surroundings is the remotely controlled power station. It is not unusual for water power to be available under just those circumstances which make the site highly unsuitable for human habitation. Under these conditions, a power station may be built without any resident engineer or operative. The place of such a resident operative is taken in part by a visiting operative who packs in at long intervals to replace and repair vital parts, but who is much more effectively replaced by automatic operation and long distance control.

In the automatic operation of a power station, one meets much the same problems which one finds in the operation of a railroad switching and signal system. There are certain things which must not be done at all costs. No generator should be connected with the bus bars until it is brought up to speed, and is running in the proper phase relations with the others. A penstock must not be thrown suddenly open in the lower end, or it will collapse under atmospheric pressure; and, on the other hand, it must not be subjected to the shock of too sudden turning on of water, or it will burst. These contingencies must be blocked in the same way as the conjunction of a closed switch and an open signal must be blocked in railroad practice.

These are relatively simple undertakings. As stated, they seem scarcely deep enough to have a mathematical form. However, behind them is a principle which is well susceptible to mathematical treatment. The principle, namely, is that the performance of a piece of apparatus should be fed back to it as information on which it is to operate.

What is more interesting to us mathematically at present, however, is not these purely local devices which modify the nature of the terminal apparatus in the generating station, and may be considered as part of the operation itself, but rather the messages which go between the load dispatcher and the station. Such messages must exist. A little cloud passes over the city to which the power is sent, and thousands of lights go on in the offices, shops and homes. This immediately demands the sending of more power; and this in turn means the starting of new turbines, the switching in of new generators, and a thousand and one other operations. Let there be an accident in the remote generating station, and even if this accident can be compensated for temporarily by automatic machinery, a message must go out to the plant engineer to send in a troubleshooter to restore the plant to maximum efficiency. In other words, the auto- 
matic substation must listen, and it must speak. Since, however, its ears and its mouth have been made by engineers for these specific purposes, and not installed by a complicated embryological process, we know far better what to expect of them than we do of human mouths and ears.

It may be remarked in passing that it is quite common practice to transmit these messages to and from the power station either over ordinary telephone lines and similar facilities, or over a carrier sent over the power line itself. In either case, they are generally coded messages like those of teletypewriters. If we wish to go to the trouble to make them vocal, all of the resources of the telephone and phonograph are at our disposal, but there is generally no particular point in this rather precious imitation of human function.

What the casual listener may miss in this account is the fact that the operation of such a system involves several stages of linguistic translation. The message from the dispatcher to the station is or should be committed to the line in the form best suitable for line transmission. On the other hand, it must appear in the station, not in the form of a coded sequence of dots and dashes, but in the form of a series of openings of switches, turnings on of valves, measurements of the speeds of motors or generators, and so on. The language of command must be translated into the language of action.

To understand this, we must realize what information is, and how it can be measured. When I send a series of dots and dashes, the problem of the information that it carries cannot be answered in terms of the message I send, taken by itself apart from all other messages. A single click may be able to do a large number of things, such as opening a sluice, connecting a generator to the bus bars, or replacing a burnt out electronic valve; but it cannot do these things differentially. It is only when I know the number of separate alternatives which must be transmitted and combined that I can determine how many different messages I must be able to send. It then appears that the significance of a signal is determined not only by that signal itself, but by the whole set of signals I might have sent in place of the actual one. In other words, significance is a property belonging to ensembles.

Another property belonging to larger and smaller ensembles is that of probability, or what is its equivalent, their Lebesgue measure. I do not intend to go into the whole question of the theoretical basis of probability, but shall only state that, under certain rather wide conditions, a smaller set of signals has a probability when taken to be part of a larger set, and that this probability is a number between 0 
and 1. Now, the probability that two independent events should happen simultaneously is the product of their individual probabilities. On the other hand, if information is to have a significance even remotely resembling its ordinary meaning, the amount of information given by two independent signals should be the sum of the amount of information which they convey separately. Under these conditions, since multiplication of probabilities corresponds to addition of information, the amount of information given by a signal can only be a constant multiple of the logarithm of its probability. Moreover, since the information is positive when the probability is less than one, the ratio between the logarithm of the probability and the amount of information must be negative.

The notion of the logarithms of probability is a familiar one for statistical mechanics. The logarithm of a probability is called entropy. In an ordinary statistical system, the entropy can increase spontaneously, but can never so decrease. In the transmission of information, we may lose information, but can never gain it, except from a new source. Thus in the translation which occurs between the message sent into the automatic station, and the operation of that station, there may be a loss of information, but there cannot be a gain. Under ideal circumstances, we attempt a coding which will correspond as nearly as possible to the actual operation of the power station, so that we are not sending in unused material, as we should for example if we were to transmit our orders by voice. Nevertheless, it is hardly to be expected that our code is so perfect that we lose no information.

Let us go from this problem to the problem of transmission of a message by voice or by eye from person to person. There is in the first instance a limitation of the rate at which information can be transmitted. It depends on the system of repeatable and recognizable signals which can be made with our vocal organs or our pen, or whatever other means we use of active communication, and which will be accepted by the air, or whatever other channel of transmission we use. I say recognizable, but I have already introduced an ambiguity thereby. Recognizable how, and by what? If I allow the recipient the full choice of any detecting and analyzing instrument which he may choose to demand, I get a certain rate of transmission of information, which is characteristic of the sender and of the means of transmission but which is not characteristic of the reception of this message by the ordinary listener or viewer. It is information of this type with which the communication engineer has been chiefly concerned in the past, as it is by all means the simplest to handle. However, when this information penetrates through the ear or the eye into those parts 
of the human nervous system which are specifically concerned with sound or sight, it undergoes a translation by a mechanism of which we know something, but not everything. As a matter of fact, it undergoes two or more translations. The first of these represent what is necessary to penetrate into the human sense organ, and through that sense organ to those parts of the brain which are in more or less permanent connection with it. The later translations interpret this superficial sensory representation into meaning, and give to what is received a more or less permanent place in memory and understanding. In the case of sound, the first translation is called phonetic and the later translations are termed semantic.

The problems of the loss of information in the two human translations, phonetic and semantic, are not at present susceptible to a direct investigation through the structure of the nervous system. They are however susceptible to an investigation which is empirical and experimental. Work of this sort has been done by the Bell Telephone Company, with special reference to the recognizability of sound as speech. The original measures of information, which were given more or less crudely, referred to the bands of frequency necessary for the formation of intelligible speech; and in other words were almost more measures of what the outside line and the air could carry, than of what the ear could translate. The reconstruction of intelligible speech from clicks and buzzes, by way of wave filters and resonators and other apparatus of the sort, shows that the loss of information between the ear and the part of the nervous system belonging to the ear is overwhelmingly great, and that a crude imitation of speech involving less than $1 / 10$ and perhaps even $1 / 100$ of the information carried over a telephone line, is enough for adequate conversation.

It was in view of this work that Dr. Wiesner of the Electronics Laboratory of MIT discussed with me the question as to whether anything could be done for a totally deaf man, to replace the missing sense of hearing in its most important employment, that of the recognition of conversation. The question was put to me by Dr. Wiesner, and the two of us were independently led to the same answer: namely, that the recognizable part of speech was so scanty, that it was not beyond our hope to replace it by the sense of touch backed up with adequate equipment. In other words, it appeared to us that it is perfectly possible to make the transition between sound in the outside air, and the semantic recognition of speech, by an artificial phonetic stage, making use of touch, and supplemented by adequate electrical tools. Having come to this conclusion, we started the detailed design of such apparatus. We took speech through an ordinary microphone. 
not dissimilar to that used as a receiver for the usual hearing aids, and quite adequate to take in all that is intelligible in speech. We then split the band received into a number of frequency bands, making use of wave filters. As we intended to use the hand as a receiver, five seemed the natural number of bands to take, because we could then carry them to the five fingers. However, we are not yet finally decided that five is the right number and may experiment on supplementing it by additional stations in the palm of the hand, so as to bring the number up to six or seven. The different bands we have so far taken to be of equal logarithmic length, or length when measured in terms of the number of keys of the piano corresponding to them. Whether this is the best mode of speech, we are not yet sure. It is a subject for further experiment.

At any rate, after receiving and separating these bands we rectify them, which means, nearly, that we replace the time-patterns of that band by its envelope. These rectified messages are then used to modulate an equal number of vibrators at frequencies of something like 100 cycles each. This is because the skin is very insensitive, both to nearby constant messages and to messages of excessively high frequency. These modulated messages are then carried either to electrical mechanical vibrators placed on the five fingers, or to electrodes designed for the direct stimulation of the nerve endings in the skin.

From the standpoint of lightness of weight, it would seem that a direct electrical stimulation is desirable. However, there are several difficulties to be overcome. The variable resistances of the skin, and the fact that current rather than voltage is what stimulates touch, both indicate that it is desirable to use constant current electrodes, which can be properly designed. However, even with these, the margin between the lower threshold of feeling and the lower threshold of pain is so small that our apparatus must work over a very narrow range, and our present models need considerable modification.

The mechanical vibrator, on the other hand, tends to be undesirable, because its current demand is at present so great as to require an apparatus which is still too heavy to be comfortably portable. We believe that whether we finally arrive at mechanical or electrical stimulators, the problem of making the apparatus portable, and indeed of comparable weight with that actually used in existing hearing aids, is not too difficult to be solved, and is more one of development than anything else.

Let me first state how our results stood when we left off work early in the summer. This work was undertaken by Dr. Leon Levine, with the cooperation of Dr. Alex Bavelas of our Psychology Department 
in matters which concerned techniques of teaching. It was desirable to see if there was in fact a sufficient basis of recognizable and distinguishable syllables in speech for us to go ahead. In the beginning, we did not experiment with the deaf, but with hearing people whose ears were filled with such a jumble of artificial noise that they were quite completely deaf for the purposes of the experiment. We then gave them a sequence of syllables by the machine accompanied with a visual pattern display of these syllables. After they had learned these, we subjected them to a series of trial syllables selected from the sequence; and we recorded the successes and failures. The best run we had was a series of 80 selections among 12 syllables with only six errors.

It has been our idea from the beginning that the learning process necessary for achieving proficiency with the new method of receiving speech must be something quite long and arduous, and we have not wished to involve any deaf mutes in an experiment which might give them a false hope, or at any rate a bad learning technique. However, we did make a certain number of experiments with a blind deaf mute, who came to us with his brother. The two had learned to communicate together by speech, which was recognized by the deaf mute when he placed his hands on his brother's larynx. The deaf mute could himself enunciate a few words, but the enunciation was very breathy and bad. After he had been put in the apparatus, he was for the first time in his life able to compare his brother's speech with his own, particularly in the enunciation of his own name and that of his brother. Within a matter of minutes, and certainly of hours, the improvement in his enunciation was not merely noticeable, but overwhelming. It was perfectly clear to us that our apparatus is an adequate way for a deaf mute, even for a blind deaf mute, to monitor his own speech.

I want to say at this point that soon after we had begun our work, we recognized very definitely that it was closely related to the visual speech developed by the Bell Telephone Company. I also wish to say that the patents which the Bell Telephone Company has based on this work were so phrased as to cover the work we had done, although our methods had not been actually employed experimentally by the Bell workers. The advantage which we believe our methods possess over visual speech, in addition to the obvious one that they can be used by blind deaf mutes, are that we have been working for portability in the apparatus. It was our conviction before we started, and it has been very greatly confirmed by our results, that the apparatus which we finally achieve should be completely portable, and 
should not deprive one of the concurrent use of such an important sense as vision. It is only by this portability that the speaker's words can be continually monitored, so that he is never allowed to fall into the faulty speech of the congenital deaf mute. We hope that, with the use of this apparatus, the speech-training of the deaf mute can be begun in early childhood, and that although it will be arduous training, it may not at that period be too different from that training which hearing children receive in learning to speak.

It will be a curious study to see what these hearing aids will do for the musical sense. They were not designed for this purpose, and I do not expect any very great results. Still, people like Helen Keller have reached a surprising degree of proficiency in recognizing musical vibrations with their fingers, and it is scarcely to be expected that the results with these machines will be worse than with the bare fingers. All in all, however, the social function of hearing is so nearly exclusively a matter of the reception of speech, that a person who can follow speech in the basis of sound carried by the air, and can do this with reasonable proficiency, can scarcely be considered socially deaf.

It is a little unfortunate that we have to use so valuable a part of the body as the hand for a receiver. We intend to use the left hand alone, and even as far as this is concerned, we intend to hold the receivers in a flexible glove, so that a gross use of the grasp by the hand is not incompatible with the simultaneous reception of speech. The trouble with the other sensitive receiving parts of the body is that the best of them lie around the mouth which must be left without impediment to participate in conversation.

It is thus conceivable at least to us that deafness as a considerable social problem might even be eliminated, except for those not mentally able to face the stringent training needed, or for those with serious concomitant physical defects. We have no such expectation for the replacement of vision.

There is reason to believe that the total amount of information received at a given time by a normal eye is of the order of 50 to 100 times what is received in the same time by a normal ear. In this, the type of measurement of vision as given by the oculist's ratio is deceptive. Vision which is $50 / 100$ means ability to recognize details at 50 feet which would ordinarily be recognized at 100 . However, the number of details varies as the square of the distance, so that from this point of view $30 / 100$ vision should give about a quarter of the amount of possible information which normal vision gives. On this basis, if the whole auditory region of the cortex were turned over to the purposes of vision, we should expect about $10 / 100$ vision. This is 
not blindness, but very poor vision indeed. Touch is an even worse surrogate for vision. Under these circumstances, it scarcely seems worth while to try to make a universal apparatus to replace vision for all purposes.

There are two directions in which progress should be expected. One of these is a reading apparatus, which will translate the pages of an ordinary book into a sound or touch pattern recognizable by the blind. The other is an aid whereby the blind man may go around more freely out of doors and in unfamiliar rooms. In the construction of an apparatus of this latter sort, there is a limitation below as well as a limitation above. Any apparatus must be better than what the blind man can do for himself by using such odds and ends of sensation as auditory echo, air temperature, air pressure of the forehead, and so forth.

Finally, besides the problem of the blind and the deaf, the problem of the maimed is also one with a profound sensory aspect which is of ten neglected. The better an artificial limb is from the standpoint of variability of performance and flexibility, the more information as to its position and as to the strains on it which the cripple needs if he is to walk securely. Similar remarks apply to artificial hands. There is a very considerable future in the art of applying strain and pressure gauges to artificial limbs, in such a way as to furnish the cripple with better sensory monitoring than he can obtain with the aid of anything but the skin of his stump. What the cripple has lost is not only motion, but sensation as well. He is an ataxic, as well as a paralytic, and anything that can be done to furnish him with sensory feedback channels other than those which he normally possesses is likely to make a great contribution to his effectiveness and to his well-being.

Massachusetts Institute of Technology 